\title{
Unsupervised clustering of multi-omics molecular layers reveals consensus molecular subtypes showing potential therapeutic opportunities for pancreatic cancer
}

\author{
Sung Hwan LEE ${ }^{* 1}$, Jiyeon PARK ${ }^{2}$, Hye Jung $\mathrm{CHO}^{1}$, Incheon KANG', Sung Hoon CHOI', Sunyoung LEE ${ }^{3}$, Ju-Seog LEE ${ }^{4}$ \\ 'Department of Surgery, CHA Bundang Medical Center, CHA University School of Medicine, Seongnam, Korea \\ ${ }^{2}$ Department of Life Sciences, McMaster University, Hamilton, ON, Canada \\ ${ }^{3}$ Department of Gastrointestinal Medical Oncology, Division of Cancer Medicine, University of Texas MD Anderson Cancer Center, Houston, TX, USA \\ ${ }^{4}$ Department of Systems Biology, Division of Basic Sciences, University of Texas MD Anderson Cancer Center, Houston, TX, USA
}

\begin{abstract}
Introduction: Pancreatic cancer is a lethal disease showing dismal prognosis and therapeutic resistance. Previous molecular subtypes from genome or transcriptome did not show clinical relevance regarding precision strategy for optimal therapeutic options followed by precise patient classification. This study aims to uncover consensus molecular subtypes from cancer-specific multi-omics data showing clinically relevant therapeutic opportunities.

Methods: We performed comprehensive analyses using the dataset from the cancer dependency map (DepMap) project, including cancer-specific molecular characterization with multi-omics data, genome-wide loss-of-function screening using the CRISPR-Cas9 system, and cancer drug sensitivity. The subtype-specific molecular signatures were validated in independent translational cohorts (TCGA-PAAD; $\mathrm{n}=150$, ICGC-PACA-AU; $\mathrm{n}=$ 461, ICGC-PACA-CA; $\mathrm{n}=317$ ).

Results: Integrative profiling of multi-omics molecular layers (Mutational signature, Copy number alteration, Transcriptome, MicroRNA, Chromatic profile, Proteome, and Metabolome) from pancreatic cancer cell lines ( $\mathrm{n}=59)$ from the Cancer Cell Line Encyclopedia (CCLE) revealed a total of three cancer-specific molecular subtypes showing distinct tumor biology through all omics layer as well as clinical relevance with unique molecular dependency. Major molecular features of each subtype were reproducible in the validation cohorts. Subtype-specific molecular biomarkers, including mutational signature and metabolites, were identified. Finally, the target drug with subtype-specific genetic dependency was analyzed to provide precision strategy according to distinct subtypes' molecular characterization.

Conclusions: Integrative profiling from multi-omics molecular layers revealed precision strategies based on cancer-specific molecular subtypes of pancreatic cancer in terms of tumor classification and discriminative therapeutic opportunities. Prospective translational studies companion with clinical trials based on cancer-specific molecular subtypes is mandatory to establish the precision strategy for managing pancreatic cancer.
\end{abstract}

